\title{
Research on the applicability of instrument rating test for textile color fastness
}

\author{
Zhilei YUAN $^{1, a}$,Yawen ZHANG $^{2, b}$,Jiashi CAI ${ }^{1, c}$ \\ ${ }^{1}$ Shanghai Entry-Exit Inspection And Quarantine Bureau, Shanghai 200135, China \\ ${ }^{2}$ College of Textile, Donghua University, Shanghai 201620, China \\ ayuanzl@shciq.gov.cn, ${ }^{b}$ yawenzhang@163.com, ${ }^{\text {c }}$ caijs@shciq.gov.cn
}

\begin{abstract}
Keywords: textiles; color fastness; instrument rating test; applicability
Abstract. In order to study the applicability of instrument rating test for different types of fabrics in color fastness experiment, spectrophotometer and digital image grading system were used to rate in different conditions for different types of fabrics .Testing conditions include color-different formulas, standard illuminations, geometric conditions and field angles. The types of fabric include napfabric,mesh fabric, coated fabrics, multicolor pattern fabric ,shiny fabric, etc. The rating results of instrument were compared with visual rating results.It is concluded that instrumental rating is available for most samples expect for yellow coated fabrics. The applicability of Digieye system is better than spectrophotometry in instrumental methods. For special fabrics, instrumental method should be combined with visual assessment to make comprehensive evaluation.
\end{abstract}

\section{Introduction}

Fastness is the resistance to external effects of color which is dyed into fabrics in the process of producing and using. It is one of the most basic and important security technical specifications ${ }^{[1]}$. The traditional method for color fastness rating is visual assessment which is relatively convenient and easy. But it is effected by subjective factors of operators and requires inspectors, rating experience. It also needs to be compared with other inspectors regularly. According to the new revised standards of test methods for color fastness, instrument rating is one of the main color fastness rating methods.Compared with visual assessment, instrument rating is of high preciseness and more objective. Instrument rating can be mainly divided into spectrophotometry and digital image technology. The former method measures the spectral power distribution 、 color density 、 chromaticity coordinates 、tristimulus values etc ${ }^{[2]}$. Digital image technology measures RGB of samples, image and calculates standard colorimetric values based on standard color cards ${ }^{[3]}$. But for the fabrics with special surface features, there are some differences between instrument rating and visual assessment results. Besides, the results are effected by test parameters which include color-different formulas, standard illuminates, geometric conditions and field angles etc. The applicability of instrument rating test for six kinds of fabrics with special surface features were analyzed by using three kinds of color measurement instruments in different testing conditions.

\section{Instrument and Test Conditions}

Digieye digital image rating system、Coloreye 7000A spectrophotometer and Hunter labscan XE colorimeter were used in the research. Digital camera with high pixel is used for capturing the images of samples. The software analyses and measures the colors with images. The color value is calculate based on CIE DE2000 color-different formulas.Spectrophotometric principle is used in coloreye 7000A spectrophotometer and Hunter labscan XE colorimeter. They measure spectral power distribution reflected by samples and calculate tristimulus values of colors in the conditions of standard illuminates and observers. The color value is calculate based on CIE LAB color-different formulas and convert to color fastness grade. There are four kinds of conditions in the process of instruments rating test. 


\section{Standard illumination}

Color is different in different illuminations. So color fastness rating test should be under the same and stable illuminates. CIE illuminate D65 and A are commonly used in textile color instrument test. Illuminates $\mathrm{A}$ is a artificial light source used inflatable tungsten of correlated color temperature of $2856 \mathrm{~K}$ to simulate the standard light source A. Standard illuminate D65 is the daylight about a correlated color temperature of $6500 \mathrm{~K}^{[4]}$.

The color temperature of standard illuminate A is lower than standard illuminate D65. The energy in long-wave of the red zone is significantly higher than the short-wave of violet region and the light color of illuminate $\mathrm{A}$ is yellowish overall ${ }^{[5]}$. In this study, illuminate D65 was used in Digieye digital image system and Hunter labscan XE colorimeter. Illuminate D65 and A were used in Coloreye 7000A spectrophotometer.

\section{Color-different Formulas}

CIE DE2000 and CIE LAB formulas are commonly used in textile color fastness rating.

\section{CIE LAB Formula}

CIE introduced CIE LAB color space and color difference formula in 1976. CIELAB formula is calculated as follows ${ }^{[6]}$ :

$$
\Delta E=\left[(\Delta L *)^{2}+\left(\Delta H_{a b} *\right)^{2}+\left(\Delta C_{\mathrm{a} b} *\right)^{2}\right]^{\frac{1}{2}}
$$

In this formula, $\Delta L^{*}, \Delta C_{a b} * \Delta H_{\mathrm{ab}} *$ is the lightness difference, saturation difference and hue difference.Although the CIE LAB color space is not completely uniform, it is easy to find the corresponding color according to the position of coordinate space.

\section{CIE DE2000 Formula}

CIE introduced CIE DE2000 based on CIE LAB in 2000. This formula is calculated as follows:

$$
\Delta E_{2000}=\left[\left(\frac{\Delta L^{\prime}}{k_{L} S_{L}}\right)^{2}+\left(\frac{\Delta C^{\prime}}{k_{C} S_{C}}\right)^{2}+\left(\frac{\Delta H^{\prime}}{k_{H} S_{H}}\right)^{2}+R_{T}\left(\frac{\Delta C^{\prime}}{k_{C} S_{C}}\right)\left(\frac{\Delta H^{\prime}}{k_{H} S_{H}}\right)\right]^{\frac{1}{2}}
$$

In this formula, $k_{L} 、 k_{C} 、 k_{H}$ is lightness 、 saturation and hue weighting parameter. For textile samples, $k_{L}=1.5, k_{C}=k_{H}=1$.

In this study, CIE LAB formula was used in Coloreye 7000A spectrophotometer and Hunter labscan XE colorimeter .CIE DE2000 formula was used in Digieye digital image system.

\section{Field Angles}

Color observed under different viewing angle will be different. The ability of distinguishing color difference is lower in the case of a small field angle(less than $4^{\circ}$ ). When field angles from $2{ }^{\circ}$ to $10^{\circ}$, the accuracy of color which match to human,s eye and the ability of distinguishing color difference is improving $^{[7]}$. But the accuracy of color matching is improved little when increase the field angle further. $2^{\circ}$ or $10^{\circ}$ is commonly used in color measurement. Its schematic diagram shown in Fig. 1.

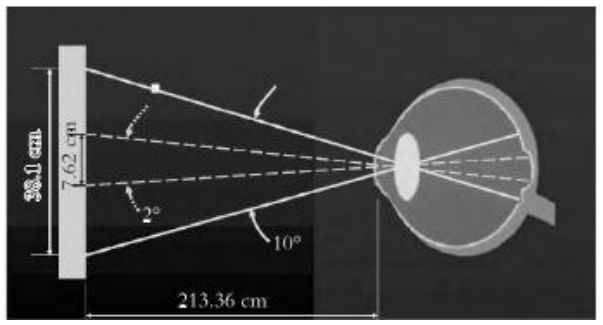

Fig. 1 schematic diagram of $2^{\circ}$ and $10^{\circ}$ field angles

In this study, $2^{\circ}$ and $10^{\circ}$ were used in Digieye digital image system. $10^{\circ}$ was used in Coloreye 7000A spectrophotometer and Hunter labscan XE colorimeter. 


\section{Geometric Conditions}

Geometric conditions refer to the geometric relationship between the illumination、 detector of color measurement instruments and test samples ${ }^{[3]}$. They mainly include $\mathrm{d} / 8 、 45 / 0$ and $0 / 45$ in textile color measurement.

$\mathrm{d} / 8$ reflects the angle between axis of reflected light beams and samples, s center normal line is $8^{\circ}$. In all directions of receiving light beams axis with $5^{\circ}$, the radiation reflected by aperture is uniform ${ }^{[8]}$. $45 / 0$ reflects the angle between axis of illumination beams and normal line of samples, surface is $45^{\circ} \pm 2^{\circ}$. In addition the angle between observation direction and normal line of samples, surface is less than $10^{\circ}$. The angle between axis of illumination beams and any beams is less than $8^{\circ[9]}$.

$0 / 45$ reflects the angle between axis of illumination beams and normal line of samples, surface is less than $10^{\circ}$. Observed samples under $45^{\circ} \pm 2^{\circ}$ from the normal line. The angle between axis of illumination beams and any beams is less than $8^{\circ}$.

In this study, $d / 8$ and 45/0 were used in Digieye digital image system. $d / 8$ was used in Coloreye 7000A spectrophotometer and 0/45 was used Hunter Labscan XE colorimeter.

\section{Experiment}

\section{Sample}

Six kinds of fabrics with special surface features were tested for color fastness. They include uneven fabrics, coated fabrics, mesh fabrics, multicolor pattern fabrics, nap fabric and shiny fabrics. Each kind fabric has five different pairs of samples.

\section{Test Standard}

Spectrophotometric refers to FZ/T 01023-1993:Method for the instrumental assessment of the degree of staining of adjacent fabrics and FZ/T 01024-1993:Instrumental assessment of change in color for determination of gray scale rating. Digieye digital image system refers to ISO 105-A11: 2012 Determination of color fastness grades by digital imaging techniques ${ }^{[10 \sim 12]}$.

\section{Data Processing Method}

The differences of results between visual assessment and instrumental assessment are expressed in terms of root mean square error(RMS)given in Eqn 3 which measures the disagreement between two sets of data.

$$
R M S=\sqrt{\frac{\sum_{i=1}^{n}\left(X_{i}-Y_{i}\right)^{2}}{n}}
$$

Where $X_{i}$ and $Y_{i}$ are the two sets of values for fastness grade. $R M S$ is smaller, the consistency between visual assessment and instrumental assessment is better. 


\section{Results and Discussion}

\section{Uneven Fabrics}

The rating results of 5 different uneven fabrics in different testing conditions are shown in Table 1.

Tab.1 rating results of uneven fabrics

Unit: grade

\begin{tabular}{|c|c|c|c|c|c|c|c|c|}
\hline No. & $\begin{array}{c}\text { digieye } \\
\text { D65/10 d/8 }\end{array}$ & $\begin{array}{c}\text { digieye } \\
\text { D65/2 d/8 }\end{array}$ & $\begin{array}{c}\text { Coloreye } 700 \\
\text { 0A D65/10 }\end{array}$ & $\begin{array}{c}\text { Coloreye70 } \\
\text { 00A A/10 } \\
\end{array}$ & $\begin{array}{c}\text { Labscan } \\
\text { XE D65/10 }\end{array}$ & $\begin{array}{c}\text { digieye } \\
\text { D65/2 45/0 }\end{array}$ & $\begin{array}{c}\text { digieye D65/10 } \\
45 / 0\end{array}$ & $\begin{array}{l}\text { visual } \\
\text { results } \\
\end{array}$ \\
\hline 1 & 1 & 1 & 1 & 1 & 1 & 1 & 1 & 1 \\
\hline 2 & 1 & 1 & 1 & 1 & 1 & 1 & 1 & 1 \\
\hline 3 & 4 & 4 & 4.5 & 4.5 & 4 & 4.5 & 4 & 4.5 \\
\hline 4 & 2 & 2 & 2.5 & 3 & 1.5 & 2 & 2 & 2 \\
\hline 5 & 2.5 & 3 & 2 & 2 & 2 & 3 & 2.5 & 2.5 \\
\hline RMS & 0.22 & 0.32 & 0.32 & 0.5 & 0.39 & 0.22 & 0.22 & \\
\hline
\end{tabular}

As can be seen from the above results, there is a good agreement between instrumental assessment and visual assessment in the conditions of illumination D65 and CIE DE2000 formula. The results of Digieye system have good consistency with visual assessment at $d / 8$ and $45 / 0$. The resulta of $10^{\circ}$ are more accurate than $2^{\circ}$ When at $\mathrm{d} / 8$. There is no big difference between the result of Digieye system and visual assessment in different visual angles when at 45/0. It is concluded Digieye system is available for assessing the color fastness of uneven fabrics used in this paper. The conditions of D65/10 ${ }^{\circ}$ standard illumination/ observer and 45/0 are suitable for this grading.

\section{Coated Fabrics}

The rating results of 5 different coated fabrics in different testing conditions are shown in Table 2.

Tab.2 rating results of coated fabrics

Unit: grade

\begin{tabular}{|c|c|c|c|c|c|c|c|c|}
\hline No. & $\begin{array}{c}\text { digieye } \\
\mathrm{d} 65 / 10 \mathrm{~d} / 8\end{array}$ & $\begin{array}{c}\text { digieye } \\
\mathrm{d} 65 / 2 \mathrm{~d} / 8\end{array}$ & $\begin{array}{l}\text { Coloreye } 70 \\
00 \mathrm{~A} \text { d65/10 }\end{array}$ & $\begin{array}{c}\text { Coloreye70 } \\
\text { 00A A/10 }\end{array}$ & $\begin{array}{c}\text { Labscan } \\
\text { XE } \\
\text { d65/10 }\end{array}$ & $\begin{array}{c}\text { digieye } \\
\mathrm{d} 65 / 245 / 0\end{array}$ & $\begin{array}{c}\text { digieye } \\
\mathrm{d} 65 / 1045 / 0\end{array}$ & visual results \\
\hline 1 & 1.5 & 1.5 & 1.5 & 1.5 & 1.5 & 2 & 2 & 1.5 \\
\hline 2 & 4 & 4 & 4 & 4 & 4 & 4 & 4 & 3.5 \\
\hline 3 & 4.5 & 4.5 & 4.5 & 4.5 & 4 & 4.5 & 4.5 & 2 \\
\hline 4 & 2.5 & 2 & 1.5 & 1.5 & 1.5 & 2.5 & 2.5 & 2.5 \\
\hline 5 & 4.5 & 4 & 4.5 & 4.5 & 4.5 & 4 & 4.5 & 4.5 \\
\hline RMS & 1.14 & 1.18 & 1.22 & 1.22 & 1.02 & 1.18 & 1.16 & \\
\hline
\end{tabular}

As can be seen from the above, results of three instrumental ratings for the third yellow coated sample is respectively $4.5 、 4.5$ and 4 . While visual result is 2 , there is a big difference between the two methods. The first、second and fifth sample is yellow-white, red and blue. The variations of these results between instrumental ratings and visual assessment are corresponds to the acceptable tolerance of half grade.The size of spectrophotometer aperture limits the grading results of yellow-white fabric with uneven discoloration. Instrumental results of the fourth fabric are less than the normal and have variations about one grade for the small test area.It is concluded instrumental 
grading is available for coated fabrics used in this research expect for yellow coated fabrics.

\section{Mesh Fabrics}

The rating results of 5 different mesh fabrics in different testing conditions are shown in Table 3.

Tab.3 rating results of mesh fabrics

\begin{tabular}{|c|c|c|c|c|c|c|c|c|}
\hline No. & $\begin{array}{c}\text { digieye } \\
\mathrm{d} 65 / 10 \mathrm{~d} / 8\end{array}$ & $\begin{array}{c}\text { digieye } \\
\mathrm{d} 65 / 2 \mathrm{~d} / 8\end{array}$ & $\begin{array}{l}\text { Coloreye } 70 \\
00 \mathrm{~A} \text { d65/10 }\end{array}$ & $\begin{array}{c}\text { Coloreye } 70 \\
\text { 00A A/10 }\end{array}$ & $\begin{array}{c}\text { Labscan } \\
\text { XE } \\
\text { d65/10 }\end{array}$ & $\begin{array}{c}\text { digieye } \\
\mathrm{d} 65 / 245 / 0\end{array}$ & $\begin{array}{c}\text { digieye } \\
\mathrm{d} 65 / 1045 / 0\end{array}$ & $\begin{array}{l}\text { visual } \\
\text { results }\end{array}$ \\
\hline 1 & 4.5 & 4.5 & 4.5 & 4.5 & 4.5 & 4 & 4 & 4.5 \\
\hline 2 & 4 & 4 & 4 & 3.5 & 3 & 4.5 & 4.5 & 4 \\
\hline 3 & 4 & 4 & 3.5 & 3.5 & 4.5 & 5 & 5 & 4.5 \\
\hline 4 & 4 & 4 & 3.5 & 3.5 & 3 & 4.5 & 4.5 & 4.5 \\
\hline 5 & 4 & 4 & 4.5 & 4.5 & 4.5 & 4.5 & 4.5 & 4.5 \\
\hline RMS & 0.39 & 0.39 & 0.63 & 0.67 & 0.81 & 0.39 & 0.39 & \\
\hline
\end{tabular}

As can be seen from the above results, the test results of Digieye system for mesh fabrics have good consistency with visual assessment and are less affected by different geometric conditions and visual angles. But the variations of these results between spectrophotometer and visual assessment are above one grade. The main reason is that color measurement is affected by the color of instrumental holding components because of mesh. While the back plane of Digieye system is gray and it can choose testing area from the images. So it is concluded that Digieye system is available for assessing the color fastness of mesh fabrics used in this research and there is a consistency between its results and visual evaluation in different geometric conditions and visual angles.

\section{Multicolor Pattern Fabrics}

The rating results of 5 different multicolor pattern fabrics in different testing conditions are shown in Table 4.

Tab.4 rating results of multicolor pattern fabrics

Unit: grade

\begin{tabular}{ccccccccc}
\hline No. & $\begin{array}{c}\text { digieye } \\
\mathrm{d} 65 / 10 \mathrm{~d} / 8\end{array}$ & $\begin{array}{c}\text { digieye } \\
\mathrm{d} 65 / 2 \mathrm{~d} / 8\end{array}$ & $\begin{array}{c}\text { Coloreye70 } \\
\text { 00A d65/10 }\end{array}$ & Coloreye70 & $\begin{array}{c}\text { Labscan } \\
\mathrm{XE}\end{array}$ & $\begin{array}{c}\text { digieye } \\
\mathrm{d} 65 / 10\end{array}$ & $\begin{array}{c}\text { digieye } \\
\mathrm{d} 65 / 2\end{array} 45 / 0$ & visual \\
$\mathrm{d} 65 / 1045 / 0$ & results \\
\hline 1 & 1.5 & 1.5 & 2 & 2.5 & 1.5 & 1.5 & 1.5 & 1 \\
2 & 1.5 & 1.5 & 1.5 & 1 & 1 & 1.5 & 1.5 & 1.5 \\
3 & 1 & 1.5 & 1 & 1 & 1 & 1.5 & 1 & 1 \\
4 & 4 & 4 & 3 & 3 & 2.5 & 4.5 & 4.5 & 4.5 \\
5 & 4 & 4 & 3 & 3 & 2.5 & 4 & 4 & 4 \\
RMS & 0.32 & 0.39 & 0.92 & 1.07 & 1.16 & 0.32 & 0.32 & \\
\hline
\end{tabular}

As can be seen from the above results, the results of Digieye system have a good agreement with visual assessment in $d / 8$ and 45/0. Spectrophotometer can only measure the color which the area of samples is bigger than testing hole because the limit of the size of testing hole. So it is hard for spectrophotometer to measure the color change of samples, small parts with complex patterns and thin strips.It is concluded that Digieye system is available for assessing the color fastness of 
multicolor pattern fabrics used in this research and the results are less affected by different geometric conditions and visual angles.

\section{Nap Fabrics}

The rating results of 5 different nap fabrics in different testing conditions are shown in Table 5 .

Tab.5 rating results of nap fabrics

\begin{tabular}{|c|c|c|c|c|c|c|c|c|}
\hline No. & $\begin{array}{c}\text { digieye } \\
\mathrm{d} 65 / 10 \mathrm{~d} / 8\end{array}$ & $\begin{array}{c}\text { digieye } \\
\mathrm{d} 65 / 2 \mathrm{~d} / 8\end{array}$ & $\begin{array}{l}\text { Coloreye } 70 \\
00 \mathrm{~A} \mathrm{~d} 65 / 10\end{array}$ & $\begin{array}{c}\text { Coloreye70 } \\
\text { 00A A/10 }\end{array}$ & $\begin{array}{c}\text { Labscan } \\
\text { XE } \\
\text { d65/10 }\end{array}$ & $\begin{array}{c}\text { digieye } \\
\mathrm{d} 65 / 245 / 0\end{array}$ & $\begin{array}{c}\text { digieye } \\
\mathrm{d} 65 / 1045 / 0\end{array}$ & $\begin{array}{l}\text { visual } \\
\text { results }\end{array}$ \\
\hline 1 & 4 & 4 & 4.5 & 4.5 & 4.5 & 3.5 & 3.5 & 4.5 \\
\hline 2 & 4 & 4 & 4.5 & 4.5 & 4.5 & 4 & 4 & 4.5 \\
\hline 3 & 4 & 4.5 & 4.5 & 4.5 & 4.5 & 4.5 & 4.5 & 4 \\
\hline 4 & 2.5 & 2.5 & 2 & 2 & 2 & 2.5 & 2.5 & 3 \\
\hline 5 & 4.5 & 4.5 & 4 & 4 & 4.5 & 4.5 & 4.5 & 4.5 \\
\hline RMS & 0.39 & 0.45 & 0.55 & 0.55 & 0.5 & 0.59 & 0.59 & \\
\hline
\end{tabular}

The following conclusions can be drawn from this research: the test results of Digieye system at $\mathrm{d} / 8$ is agreeable with visual evaluation. There is a variation between the results of spectrophotometer and visual assessment for the surface morphology of samples are changed by the pressing force from the spectrophotometers, holding parts on nap fabrics . Therefore, Digieye system with the conditions of $\mathrm{d} / 8$ and $\mathrm{D} 65 / 10^{\circ}$ is available for assessing the color fastness of nap fabrics used in this research.

\section{Shiny Fabrics}

The rating results of 5 different shiny fabrics in different testing conditions are shown in Table 6 .

Tab.6 rating results of shiny fabrics

\begin{tabular}{|c|c|c|c|c|c|c|c|c|}
\hline No. & $\begin{array}{c}\text { digieye } \\
\mathrm{d} 65 / 10 \mathrm{~d} / 8\end{array}$ & $\begin{array}{c}\text { digieye } \\
\mathrm{d} 65 / 2 \mathrm{~d} / 8\end{array}$ & $\begin{array}{l}\text { Coloreye } 70 \\
00 \mathrm{~A} \text { d65/10 }\end{array}$ & $\begin{array}{c}\text { Coloreye } 70 \\
00 \mathrm{~A} \mathrm{~A} / 10\end{array}$ & $\begin{array}{c}\text { Labscan } \\
\text { XE } \\
\text { d65/10 }\end{array}$ & $\begin{array}{c}\text { digieye } \\
\mathrm{d} 65 / 245 / 0\end{array}$ & $\begin{array}{c}\text { digieye } \\
\text { d65/10 45/0 }\end{array}$ & $\begin{array}{l}\text { visual } \\
\text { results }\end{array}$ \\
\hline 1 & 4 & 4 & 4.5 & 4.5 & 3.5 & 4 & 4 & 4.5 \\
\hline 2 & 3 & 3 & 2.5 & 2.5 & 2.5 & 3.5 & 3.5 & 3 \\
\hline 3 & 4 & 4 & 4 & 4 & 3 & 4.5 & 4.5 & 3.5 \\
\hline 4 & 4.5 & 4.5 & 5 & 5 & 5 & 4.5 & 4.5 & 4.5 \\
\hline 5 & 4 & 4 & 4 & 4 & 4 & 4 & 4 & 4 \\
\hline RMS & 0.32 & 0.32 & 0.39 & 0.39 & 0.59 & 0.54 & 0.54 & \\
\hline
\end{tabular}

Different geometric conditions can bring the difference of receiving flux from the detector when testing the fabrics with high gloss. So geometric conditions have great effect on the color measurement. It can be seen from the above that the results of Digieye system and coloreye7000A have good consistency with view at $\mathrm{d} / 8$. While there is a diffidence above a half grade between Labscan XE at 0/45and Digieye system at $45 / 0$ with visual results. So in this research it is suitable to test the color fastness of good gloss fabric by Digieye system and coloreye $7000 \mathrm{~A}$ at $\mathrm{d} / 8$. 


\section{Conclusion}

Through experimental analysis of different surface characteristics of the fabrics, it is concluded that instrumental rating is available for most samples expect for yellow coated fabrics. The applicability of Digieye system is better than spectrophotometry in instrumental methods.Digieye system is suitable for assessing the color fastness of mesh fabrics, multicolor pattern fabrics and nap fabrics. $\mathrm{d} / 8$ geometric condition is available for testing nap fabrics and shiny fabrics. Therefor, surface features of fabrics should be considered and choosing the appropriate test equipment and conditions when using instrumental method to evaluate color fastness.For special fabrics, instrumental method should be combined with visual assessment to make comprehensive evaluation.

\section{Reference}

[1]Hongxia ZHANG.Textile testing practices[M].Beijing: China Textile Press,2007:82.

[2] Wei ZHOU,Lei ZHONG,Weidong LI , etal. The correlation between visual assessment and instrumental assessment of color fastness[J].Shanghai Textile Science \& Technology, 2013, 41 (5) : $4-5$.

[3] Xiaoke JIN,Qizheng LI,Shengcheng ZHANG, etal.Classification and development of the fabric color measurement methods[J]. Journal of Textile Research, 2012（9）: 105.

[4] GB/T3978-2008, Standard illuminants and geometric conditions[S].

[5]Hongmei DU.Optical influence of illuminants D65 and $\mathrm{A}$ on the evaluation of jadeite(green)[D].Beijing, China University of Geoscience, 2010: 44.

[6] GB/T8424.3-2001, Textiles- Tests for colour fastness-Calculation ofcolour difference[S].

[7]Xiurong LI . Issues should be noted in color measurement[J]. Guangdong Printing, 2010 (1) :25.

[8] Yaoxing JIANG.Textile Laboratory Science[M].Beijing: China Textile Press,2008:93-94.

[9] Ruqin LI, Juncai SONG.Fiber and textile testing technology[M].Shanghai, Donghua University Press, 2005: 207.

[10] FZ/T 01023-1993: Method for the instrumental assessment of the degree of staining of adjacent fabrics[S].

[11] FZ/T 01024-1993:Instrumental assessment of change in colour for determination of grey scale rating $[\mathrm{S}]$.

[12] ISO105-A11:2012 Determination of color fastness grades by digital imaging techniques[S]. 